\title{
Interference with PSMB4 Expression Exerts an Anti-Tumor Effect by Decreasing the Invasion and Proliferation of Human Glioblastoma Cells
}

\author{
Yu-Chen Cheng ${ }^{a}$ Wen-Chiuan Tsaib Yu-Chi Sung ${ }^{c}$ Hsin-Han Chang ${ }^{d}$ \\ Ying Chen ${ }^{\mathrm{a}, \mathrm{d}}$ \\ aDepartment of Biology and Anatomy, National Defense Medical Center, Taipei, bepartment of \\ Pathology, Tri-Service General Hospital, National Defense Medical Center, Taipei, 'Department of \\ Medicine, National Defense Medical Center, Taipei, ' ${ }^{\mathrm{G}}$ Graduate Institute of Life Science, National Defense \\ Medical Center, Taipei, Taiwan
}

\section{Key Words}

Psmb4 • Invasion • Proliferation • Apoptosis • Glioblastoma

\begin{abstract}
Background/Aims: Glioblastoma (GBM) is a malignant brain tumor with a poor prognosis. Proteasome subunit beta type-4 (PSMB4) is an essential subunit that contributes to the assembly of the $20 \mathrm{~S}$ proteasome complex. However, the role of PSMB4 in glioblastomas remains to be clarified. The aim of this study was to investigate the role of PSMB4 in GBM tumor progression. Methods: We first analyzed the PSMB4 protein and mRNA expression in 80 clinical brain specimens and 77 datasets from the National Center for Biotechnology Information (NCBI) Gene Expression Omnibus (GEO) database. Next, we inhibited the PSMB4 expression by siRNA in cellular and animal models to explore PSMB4's underlying mechanisms. The cell survival after siPSMB4 transfection was assayed by MTT assay. Annexin V and propidium iodide staining was used to monitor the apoptosis by flow cytometric analysis. Moreover, the migration and invasion were evaluated by wound healing and Transwell assays. The expression of migration-related and invasion-related proteins after PSMB4 inhibition was detected by Western blotting. In addition, an orthotropic xenograft mouse model was used to assay the effect of PSMB4 knockdown in the in vivo study. Results: Basis on the results of bioinformatics study, glioma patients with higher PSMB4 expression had a shorter survival time than those with lower PSMB4 expression. The staining of clinical brain tissues showed elevated PSMB4 expression in GBM tissues compared with normal brain tissues. The PSMB4 inhibition decreased proliferation, migration and invasion abilities in human GBM cells. Downregulated PSMB4 resulted in cell cycle arrest and apoptosis in vitro. In an orthotropic xenograft mouse model, the glioma tumors progression was reduced when PSMB4 was down-regulated. The
\end{abstract}


decreased PSMB4 enhanced the anti-tumor effect of temozolomide (TMZ) on tumor growth. In addition, the absence of PSMB4 decreased the expression of phosphorylated focal adhesion kinase and matrix metallopeptidase 9 in vivo. Conclusion: PSMB4 inhibition in combination with TMZ may exert an anti-tumor effect by decreasing cell proliferation and invasion as well as by promoting apoptosis in human glioblastoma cells. This research may improve the therapeutic efficacy of glioblastoma treatment.

(C) 2018 The Author(s)

Published by S. Karger AG, Basel

\section{Introduction}

Glioblastoma, also known as glioblastoma multiforme (GBM), is the most lethal primary brain cancer in humans [1]. According to the World Health Organization (WHO) grading system, GBM is classified as a grade IV astrocytoma composed of poorly differentiated neoplastic astrocytes that exhibit aggressive proliferation and invasive properties $[1,2]$. Because more than $90 \%$ of brain tumor patients die within 3 months after an untreatable diagnosis, it is difficult to define which factors are in connection with tumor growth [3]. To date, the median survival of glioblastoma patients remains less than 15 months, and the 5-year relative survival rate is still less than 5\% [4-6]. Therefore, identifying the critical oncogenes and underlying mechanism involved in brain tumor growth is an extremely important task for GBM treatment.

Proteasome subunit beta type-4 (PSMB4), also known as 20S proteasome subunit beta-7, is one of the essential subunits that contributes to the complete assembly of the 20S proteasome complex [7]. PSMB4 is highly expressed in multiple cancer types, such as myeloma [8], hepatocellular carcinoma [9], ovarian cancer [10] and prostate cancer [11]. In epithelial ovarian cancer, PSMB4 expression is higher than that in normal ovary tissues and is positively related to poor prognosis and clinical pathologic variables [10]. PSMB4 expression not only enhances the lung cancer cell survival rate but also significantly facilitates tumor growth in NIH/3T3 tumor-bearing mice [12]. In recent studies using pharmacological inhibition and RNA interference, PSMB4 has been found to significantly enhance survival of the T98G glioma cell line [13]. Thus, the performance of PSMB4 in glioma may be related to tumor progress.

The aim of this study was to identify the role of PSMB4 in GBM progression. A search of National Center for Biotechnology Information (NCBI) Gene Expression Omnibus (GEO) database revealed higher expression of PSMB4 mRNA and a shorter survival time in highgrade gliomas than in low-grade gliomas. The results of immunohistochemical (IHC) staining of a clinical brain tissue microarray indicated increased PSMB4 expression in high-grade gliomas, thus suggesting that PSMB4 expression correlates with poor prognosis in highgrade gliomas. We further blocked PSMB4 in GBM cells via siRNA transfection and examined the anti-tumor effects both in vitro and in vivo. The PSMB4 deficiency greatly limited the migration and invasion ability of human GBM cells. The tumor size also substantially decreased with decreased PSMB4 expression in an orthotopic xenograft animal model. Moreover, the absence of PSMB4 strongly decreased glioma tumor invasion by decreasing the expression of phosphorylated focal adhesion kinase (p-FAK) and matrix metallopeptidase 9 (MMP-9). In summary, the 26S proteasomal subunit PSMB4 is involved in GBM progression and may potentially serve as an adjuvant therapeutic target in glioma treatment.

\section{Materials and Methods}

Analysis of human glioma datasets from the GEO database

The genomic data sets obtained from the NCBI GEO database were analyzed with methodology developed by Dr. Hueng and colleagues [14, 15]. Briefly, 100 sets of de-linked data (GDS1815/202243_s_ at) on PSMB4 mRNA expression, age, pathologic grading of primary glioma and overall survival time were obtained from http://www.ncbi.nlm.nih.gov/geo/tools/profileGraph.cgi?ID=GDS1815:202243_s_at. 


\section{Cellular Physiology Cell Physiol Biochem 2018;45:819-831

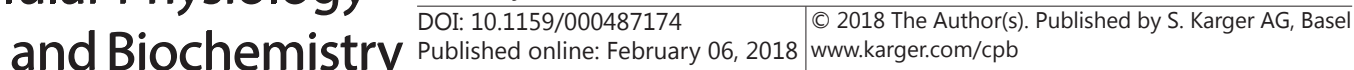 \\ Cheng et al.: PSMB4 Inhibition Reduces Glioblastoma Progression}

However, 23 data sets without thorough information on the survival period and age were excluded; thus, 77 data sets were used in the statistical analyses.

\section{Immunohistochemical staining of human glioma specimens}

A tissue microarray (GL807a; Biomax, Rochester, NY, USA) was incubated with a monoclonal rabbit anti-human PSMB4 antibody (Cat. No. ab137067, Abcam, Cambridge, UK) at room temperature, incubated with biotin-labeled secondary immunoglobulin (1:100, DAKO, Glostrup, Denmark) at room temperature, and treated with 3-amino-9-ethylcarbazole substrate chromogen (DAKO) at room temperature to visualize peroxidase activity.

\section{Cell culture}

The human LN229 and U87MG glioblastoma cell lines and GBM8401 primary cell line was provided by Dr. Hueng from the National Defense Medical Center, Taiwan. LN229-Luc2 cells were derived from stable transfection of pLuc2-iRFP and were selected via a FACS Aria Fusion Sorter. The cells were grown in Dulbecco's Modified Eagle's Medium (DMEM) containing 10\% fetal bovine serum and $100 \mathrm{IU} / \mathrm{ml}$ penicillin and streptomycin (pH 7.4) (all obtained from Gibco) in a humidified atmosphere of $5 \% \mathrm{CO}_{2}$ at $37^{\circ} \mathrm{C}$.

\section{Drugs}

The 2.3.3-[4, 5-dimethylthiazol-2-yl]-2, 5-diphenyltetrazolium bromide (MTT), propidium iodide (PI) and MG132 used in this study were obtained from Sigma-Aldrich (Sigma). Temozolomide (TMZ) was purchased from MedChem Express (MCE).

\section{siRNA Transfection}

The human LN229 and U87MG glioblastoma cell lines were transfected with $50 \mathrm{nM}$ of the following Pre-designed siRNA Products (Ambion) targeting the region of the PSMB4 mRNA (siRNA: 5'-AACCCUUUGUGGAACACCA-3' ; 5'-UGGUGUUCCACAAAGGGUU-3') or control siRNA via the RNAi-MAX Lipofectamine reagent (Invitrogen). The medium was replaced with DMEM containing 10\% fetal bovine serum after $24 \mathrm{~h}$ transfection, then culture for an additional 24, 48, 72 and $96 \mathrm{~h}$ before subsequent experiments.

Cell survival assay

LN229 and U87MG glioblastoma cells were cultured at $2 \times 10^{4}$ cells per well in a 24 -well plate. Different concentrations of drugs or vehicle control were then added to the culture medium for 24 hours. After the cells were washed with phosphate-buffered saline (PBS) $\left(137 \mathrm{mM} \mathrm{NaCl}, 2.7 \mathrm{mM} \mathrm{KCl}, 1.5 \mathrm{mM} \mathrm{KH}_{2} \mathrm{PO}_{4}, 8 \mathrm{mM}\right.$ $\mathrm{Na}_{2} \mathrm{HPO}_{4}$, and pH 7.4), $0.5 \mathrm{mg} / \mathrm{ml}$ of MTT was added, and incubation was continued for another 4 hours. The cells were then lysed with DMSO (Sigma). The absorbance at $590 \mathrm{~nm}$ was measured.

\section{Cell migration assays}

Cell migration was assayed via Transwell and wound healing assays. The wound-healing migration assay was prepared by seeding $1 \times 10^{6} \mathrm{U} 87 \mathrm{MG}$ or LN229 glioma cells in the $3.5-\mathrm{cm}$ cultured dish to form a monolayer. The cells were then cultured for $16 \mathrm{~h}$ after being scratched with a P200 pipette tip and photographed, and the migration ability results were representative of three different experiments and were analyzed with ImageJ. The Transwell migration assay was prepared by seeding $8 \times 10^{4}$ LN229 or U87MG glioma cells in the upper chamber of a Transwell (Costar). After incubation at $37^{\circ} \mathrm{C}$ for $16 \mathrm{hrs}$, the cells on the lower side were fixed with formalin and stained with Coomassie Brilliant Blue G250 (Sigma). The migrated cells were counted in three randomly selected fields from each membrane three times per group in each experiment.

\section{Cell invasion assay}

The Transwell invasion assay was prepared by seeding $4 \times 10^{4}$ U87MG or LN229 glioma cells in the upper chamber of a Transwell. Before cell seeding, $0.5 \%$ Matrigel in a coating buffer solution (BD) was added to the upper chamber and then incubated at $37^{\circ} \mathrm{C}$ for $16 \mathrm{hrs}$. The cells on the lower side were fixed with formalin and were stained with Coomassie Brilliant Blue G250. The invaded cells were counted in three randomly selected fields from each membrane three times per group in each experiment. 


\section{Cellular Physiology Cell Physiol Biochem 2018:45:819-831

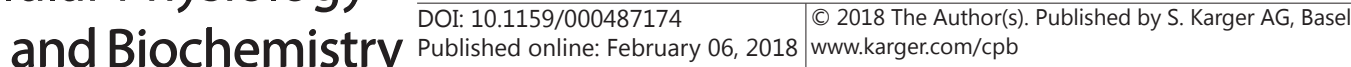

Cheng et al.: PSMB4 Inhibition Reduces Glioblastoma Progression

\section{Flow cytometric analysis}

The cell cycle and apoptosis assays were prepared by seeding $2 \times 10^{5}$ LN229 or U87MG glioma cells in 6-well plates. After cell attachment, siRNA transfection was applied for 96 hours. For cell cycle assays, cells were fixed and stained with PI. For apoptosis assays, cells were harvested and processed for Annexin $\mathrm{V}$ staining according to the manufacturer's instructions (Biovision). The cells were washed with binding buffer [4-(2-hydroxyethyl)-1-piperazineethanesulfonic acid, $140 \mathrm{mmol} / \mathrm{l} \mathrm{NaCl}$, and $5 \mathrm{mmol} / \mathrm{l} \mathrm{CaCl}_{2}$ at $\mathrm{pH}$ 7.4] and stained with anti-Annexin $V$ antibody and then counterstained with PI at room temperature. The results were measured using a FACSVerse laser flow cytometric analysis system (Becton Dickinson). Ten thousand cells were analyzed for each sample.

\section{Real-time polymerase chain reaction analysis}

Total RNA was reverse transcribed to cDNA with a Reverse Transcription kit (Invitrogen). The Applied Biosystems 7500 Fast Real-Time PCR System was used to quantify mRNA with SYBR Green ER ${ }^{\mathrm{TM}}$ SuperMix Universal (Invitrogen) according to the protocol provided by the manufacturer. The following oligonucleotide primers were used for real-time polymerase chain reaction (forward/reverse sequences): PSMB4 (5'ACTGGTTATGGTGCATACTTG/TTGTAAGAACGGGCATCTC'), MMP-2 (5'CTTCCAAGTCTGGAGCGATGT/ TACCGTCAAAGGGGTATCCAT3'), MMP-9 (5'GGGACGCAGACATCGTCATC/TCGTCATCGTCGAAATGGGC3') and GAPDH (5'AGCCACATCGCTCAGACACC/GTACTCAGAGGCCAGCATCG3'). The GAPDH gene was used as an endogenous control.

\section{Western blotting}

After the various treatments, the glioma cells were homogenized in lysis buffer (10 mM EGTA, 2 mM $\mathrm{MgCl}_{2}, 60 \mathrm{mM}$ PIPES, $25 \mathrm{mM}$ HEPES, $0.15 \%$ Triton X-100, $1 \mu \mathrm{g} / \mathrm{ml}$ of pepstatin A, $1 \mu \mathrm{g} / \mathrm{ml}$ of leupeptin, 1 $\mathrm{mM} \mathrm{NaF}$, and $1 \mathrm{mM}$ phenylmethylsulfonyl fluoride). Protein samples (60 $\mu \mathrm{g}$ per lane) were electrophoresed on a $10 \%$ SDS polyacrylamide gel and then transferred to a nitrocellulose membrane (Bio-Rad). Strips from the membrane were blocked with $5 \%$ non-fat milk in Tris-buffered saline, $\mathrm{pH} 7.4$, containing $0.1 \%$ Tween (TBS-Tween) and were incubated overnight at $4{ }^{\circ} \mathrm{C}$ with a 1:500 dilution of mouse antibodies against human phosphorylated FAK (p-FAK), FAK, phosphorylated paxillin, paxillin, integrin $\beta 1$ and integrin $\beta 3$ (Abcam) or a rabbit antibody against human PSMB4 antibody (Abcam), matrix metallopeptidase 2 (MMP-2), MMP-9, GAPDH (BioVision) and Ki67 (Genetex). After being washed, the strips were incubated with a 1:5000 dilution of HRP-conjugated anti-rabbit or anti-mouse IgG antibodies (Promega). Next, the blots were reacted in the ECL substrate developing solution (Bio-Rad). The density of the bands on the nitrocellulose membrane was quantified by densitometry using Gel Pro 3.1 (Media Cybernetics), taking the density of the control sample as $100 \%$ and expressing the density of the test sample relative to the expression of the internal control as a relative value. Phosphorylated proteins were normalized to the total protein first.

\section{Animal xenograft model}

All mouse experiments were approved by the laboratory animal center of National Defense Medical Center, Taiwan (IACUC No. 16-275). BALB/cAnN.Cg-Foxn1nu/CrlNarl nude mice (20-25 g) were anesthetized with $\mathrm{O}_{2}$ /isoflurane mixture. Next, $10^{5}$ LN229-Luc2 cells with knockdown of PSMB4 or a scrambled control were implanted into the right cerebral hemisphere of the mice. Five days after implantation, the mice were assigned to four groups that received vehicle control $(n=4)$, TMZ $(n=4)$, siPSMB4 $(n=5)$, or siPSMB4+TMZ $(n=5)$ treatments. TMZ was administered via oral gavage at $5 \mathrm{mg} / \mathrm{kg} /$ day for 7 days. Animals were euthanized using $\mathrm{CO}_{2}$ after 12 days, brains were fixed in formalin then embedded in paraffin, and serial sections. The bioluminescence intensity of the implanted tumors was monitored with a non-invasive In vivo Imaging System (IVIS) three times per week. The body weights of the mice were measured three times per week.

\section{Histological and immunohistochemistry examination}

Orthotopic tumors were excised, rinsed twice in PBS and fixed in 4\% paraformaldehyde. Tissues were frozen and sliced into $5-\mu \mathrm{m}$-thick sections. Routine hematoxylin and eosin (HE) staining was performed to facilitate histological evaluation. The expression of Ki-67, p-FAK and MMP9 in the brain tumors of the nude mice was detected by IHC. Ki-67 (Genetex), p-FAK and MMP9 (Abcam) primary antibodies and secondary goat anti-rabbit antibodies (Jackson Laboratories) were used. The expression of Ki-67, p-FAK and MMP9 was observed in 10 random fields for each group. 


\section{Cellular Physiology Cell Physiol Biochem 2018;45:819-831 and Biochemistry DOI: 10.1159/000487174 2018 (0) 2018 The Author(s). Published by S. Karger AG, Basel \\ Cheng et al.: PSMB4 Inhibition Reduces Glioblastoma Progression}

Statistical analysis

The overall survival of data sets (GDS1815/202243_s_at/PSMB4) gathered from the GE0 was analyzed by the Kaplan-Meier method. A single tailed test was used to calculate the PSMB4 expression level in the groups of WHO pathologic grade gliomas. Cohorts with low- versus high-PSMB4 gene expression were estimated with both WHO grade III and grade IV human glioma groups. The cut-off value of PSMB4 expression was based on the conditional inference tree via 'party' package with $\mathrm{R}$ language (R 3.1.2 software). All experiments were performed at least three times, and the results are expressed as the means \pm SEM for the total number of experiments. Differences between means were assessed using the KruskalWallis test. The Mann-Whitney test was used for post-hoc analysis. Statistical significance was set at $\mathrm{p}<0.05$.

\section{Results}

The increase in PSMB4 $m R N A$ and protein expression is correlated with poor survival time in human high-grade gliomas

To investigate the role of PSMB4 in human glioma, the genomic data sets from the GEO database were used to analyze the correlation of the PSMB4 expression with overall survival in human high-grade gliomas. As shown in Fig. $1 \mathrm{~A}$ and $1 \mathrm{~B}$, the survival time of 77 high-grade glioma patients revealed that patients with low PSMB4 mRNA expression $(n=50)$ had better overall survival than those with high PSMB4 mRNA expression $(\mathrm{n}=27)(P=0.0005$, by log-rank test; $95 \%$ CI: $1.620-4.959$, ratio 2.167, cut-off value set at 2384.9). The median survival times for the high- versus low-PSMB4 expression groups were 62 weeks and 124 weeks, respectively. In addition, IHC staining of human glioma tissue and normal brain tissue microarrays was conducted (Fig. 1C). The PSMB4 score of immunostaining was significantly

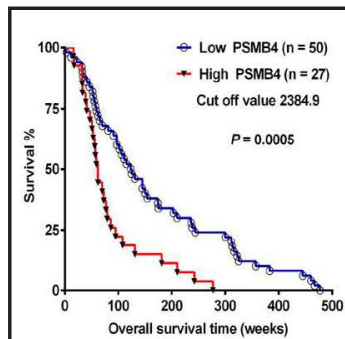

\section{A \\ B}

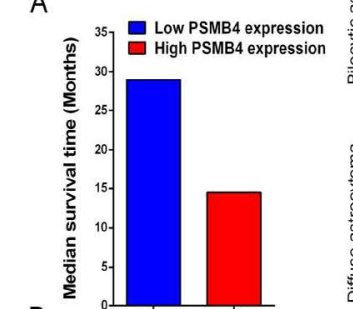

Non-neoplastic brain tissues - Low grade glioma
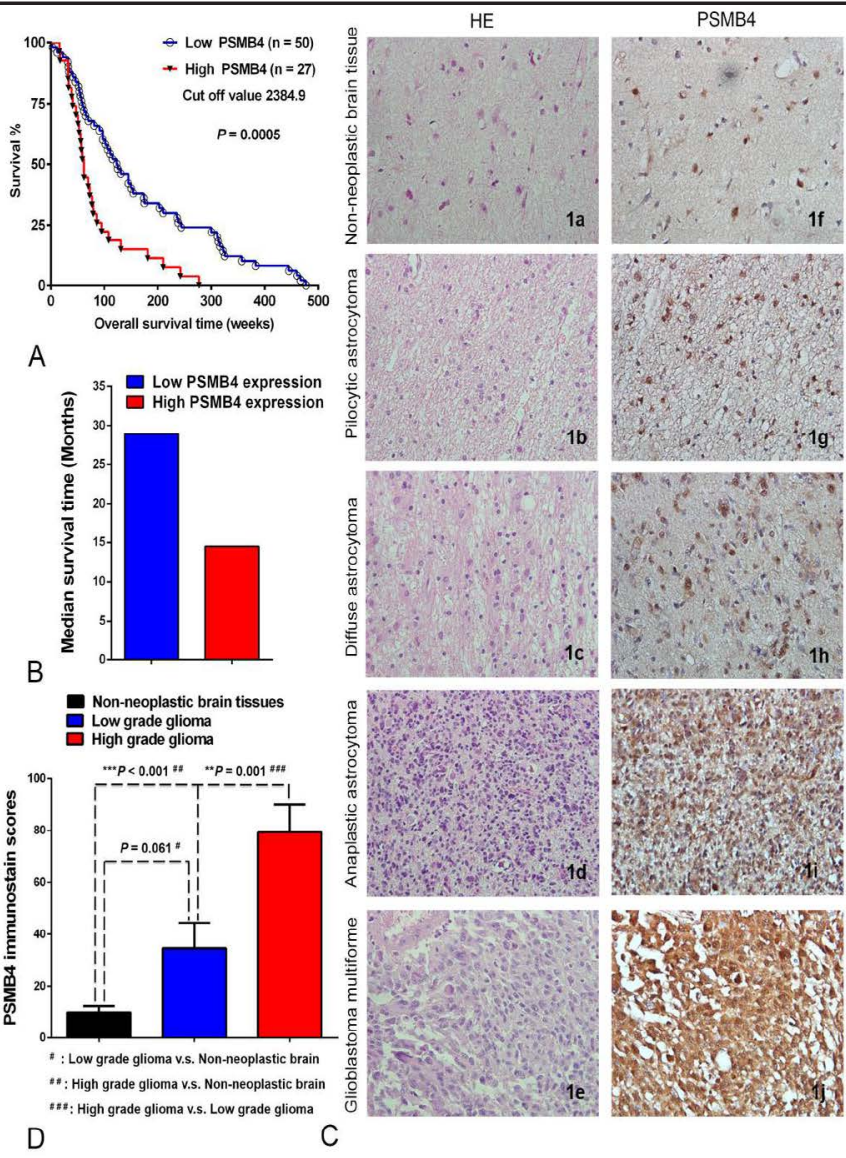

Fig. 1. PSMB $4 \mathrm{mRNA}$ and protein expression in human gliomas. (A) The up-regulation of PSMB4 mRNA expression decreases the survival time in human high-grade gliomas. The KaplanMeier survival curve shows shorter survival in those with high PSMB4 (> 2384.9) $(n=27)$ than in those with low PSMB4 $(<$ 2384.9) ( $\mathrm{n}=50)$ expression levels $(\mathrm{P}=0.0005$, by log-rank test, hazard ratio: 2.167, 95\% CI: 1.620-4.959). (B) Median survival time with high PSMB4 expression and low PSMB4 expression (median survival, 62 vs. 124 weeks). (C) PSMB4 overexpression in high-grade gliomas compared with that in low-grade gliomas and non-tumor controls. Representative hematoxylin and eosin staining of $1 \mathrm{a}$. normal brain, $1 \mathrm{~b}$. pilocytic astrocytoma, 1c. diffuse astrocytoma, 1d. anaplastic astrocytoma, and 1e. glioblastoma multiforme. Representative immunohistochemical staining of PSMB4 in 1f. normal brain, 1g. pilocytic astrocytoma, 1h. diffuse astrocytoma, 1i. anaplastic astrocytoma, and $1 \mathrm{j}$. glioblastoma multiforme. Original magnification $\times 400$. (D) Statistical analysis showed that the PSMB4 immunostaining score was significantly higher in high-grade (WHO grade III/ IV) gliomas than in lowgrade (WHO grade I/ II) gliomas, and normal brain tissue ( $\mathrm{P}=$ $0.001, \mathrm{P}<0.001$, respectively). 


\section{Cellular Physiology and Biochemistry}

Cell Physiol Biochem 2018;45:819-831

\begin{tabular}{l|l}
\hline DOI: $10.1159 / 000487174$ & (C) 2018 The Author(s). Published by S. Karger AG, Basel
\end{tabular}

Cheng et al.: PSMB4 Inhibition Reduces Glioblastoma Progression higher in high-grade (WHO grade III/IV) gliomas than in low-grade (WHO grade I/ II) gliomas or normal brain tissue (Fig. 1D, Table 1). These data demonstrated that high PSMB4 mRNA and protein expression were correlated with poor prognosis in human gliomas.

\section{Proteasome inhibitor treatment decreases cell migration in human glioblastoma cells \\ Compared with differ-} ent glioblastoma cell lines, LN229 showed significantly higher expression of PSMB4 (Fig. 2A). Before examination of the effects of PSMB4, the role of proteasome activity in cell viability was first identified by the MTT assay. After treatment with the proteasome inhibitor MG132 for 24 hours, the cell survival rates of LN229 and U87MG cells were unaffected until the concentration was greater than 200 nM (Fig. 2B). Apart from cell survival, cell migration was assessed through wound healing and the Transwell migration assays. As shown in Fig. 2C and 2D, the cell migration ability of LN229 and U87MG decreased significantly in the presence of $100 \mathrm{nM}$ MG132 treatment compared with control treatment.

Decreased expression of PSMB4 decreases cell survival and proliferation in human glioblastoma cells

After transfection with the siRNA of PSMB4, the mRNA expression of PSMB4 was significantly decreased in LN229 and U87MG cells
Table 1. PSMB4 immunostain scores in non-neoplastic brain tissue and various grades of gliomas in the tissue array. * The correlation was analyzed by Pearson Product Method Correlation test

\begin{tabular}{lccccc}
\hline & Number of cases & $\begin{array}{c}\text { Average } \\
\text { intensity }\end{array}$ & $\begin{array}{c}\text { Average } \\
\% \text { tumor }\end{array}$ & $\begin{array}{c}\text { Average } \\
\text { score }\end{array}$ & Correlation* \\
\hline $\begin{array}{l}\text { Non-neoplastic brain tissue } \\
\text { Classification of gliomas }\end{array}$ & 10 & 0.9 & 10 & 10 & \\
WHO grade I & 12 & 1 & 20 & 20 & \\
WHO grade II & 18 & 1.28 & 25.83 & 44.17 & Positive correlation \\
WHO grade III & 18 & 1.22 & 25.56 & 44.44 & $\left(\mathrm{P}=5.5 \times 10^{-5}\right)$ \\
WHO grade IV & 22 & 1.77 & 57.73 & 107.73 & \\
\hline
\end{tabular}

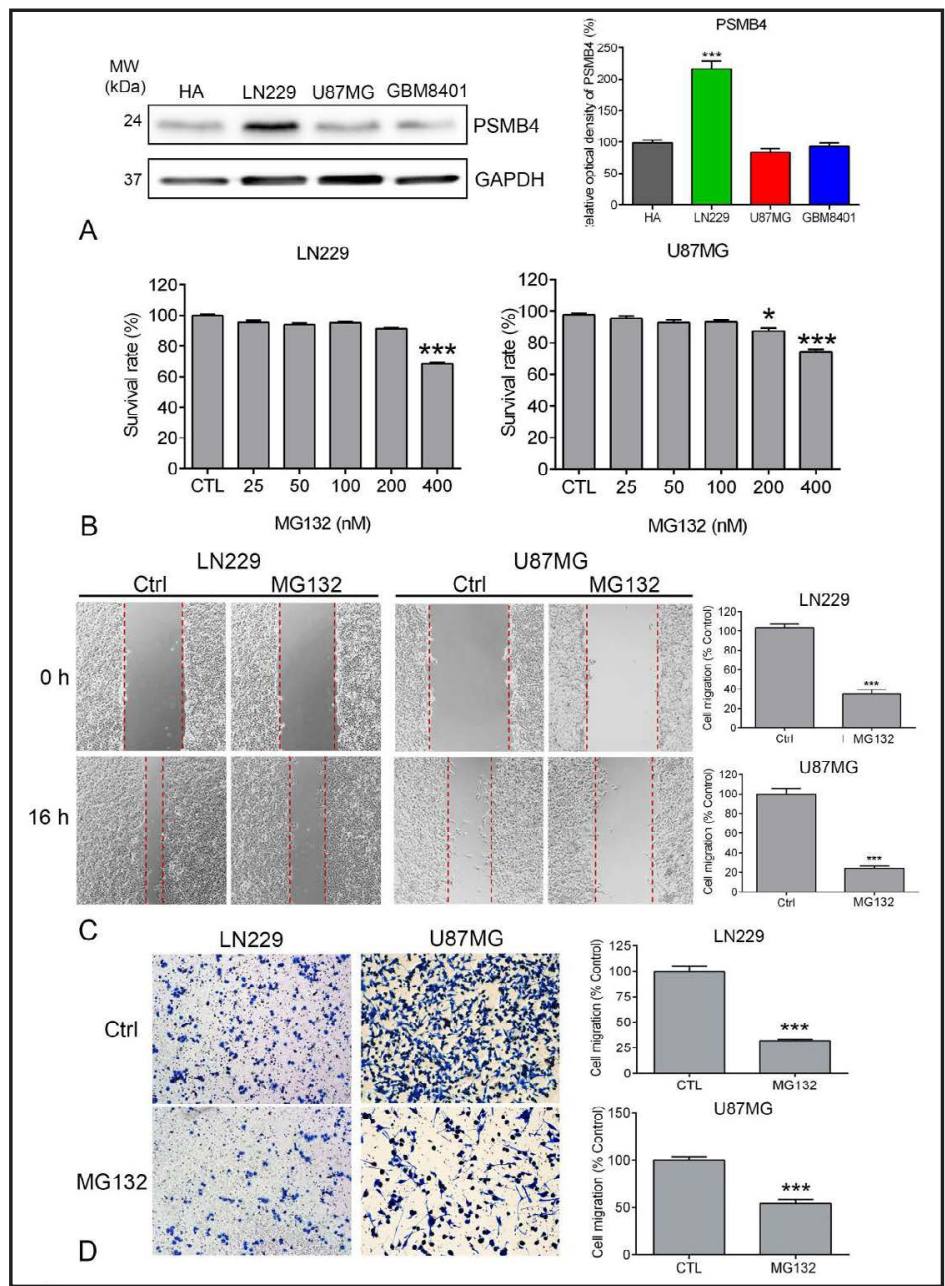

Fig. 2. Effects of proteasome inhibitor MG132 treatment on human glioblastoma cell mobility. (A) PSMB4 expression in human normal astrocytes (HA), LN229, U87MG and GBM8401. The right panels show the quantitative analyses of PSMB4 expression. ${ }^{* *} \mathrm{p}<0.001$ compared with the HA group. (B) LN229 and U87MG glioma cells were treated, respectively, with PBS and different concentrations of MG132 (25, 50, 100,200 and $400 \mathrm{nM}$ ) for 24 hours. The survival rates were analyzed with the MTT assay. The right panels show the quantitative analyses of the MTT assays. The MG132-mediated cell migration inhibition was analyzed using (C) wound healing migration assays and (D) Transwell assays. The right panels show the quantitative analyses of the wound healing and Transwell migration assays. ${ }^{*} \mathrm{p}<0.05$; ${ }^{* * *} \mathrm{p}<0.001$ compared with the control group. 
from 24 to 96 hours (Fig. 3A). In addition, the PSMB4 protein expression of LN229 and U87MG cells with siPSMB4 transfection showed a marked decrease after 48 hours. Moreover, after downregulation of PSMB4 for 96 hours, Ki67 expression was reduced in LN229 and U87MG cells (Fig. 3B). After decreased expression of PSMB4 for 72 and 96 hours, the survival rate notably declined in the siPSMB4 group compared with the control group (Fig. 3C).

Downregulation of PSMB4 arrests the cell cycle and induces apoptosis in human glioblastoma cells

To assess the cell cycle regulatory mechanisms of PSMB4 in cell growth inhibition, the PSMB4 expression of LN229 and U87MG cells was decreased by siRNA for 96 hours. As shown in Fig. 3D and Fig. 4A, downregulated PSMB4 expression resulted in decreased levels of G0/ G1 population and increased the cell population at the sub-G1 phase in both LN229 and U87MG cells. Moreover, Annexin $\mathrm{V}$ and PI staining showed that the long-term inhibition of PSMB4 protein expression promoted apoptosis in LN229 and U87MG cells (Fig. 4B-C). This finding suggested that PSMB4 is involved in the decreased survival in glioma cells, and the constant deficiency in PSMB4 expression leads to apoptosis in LN229 and U87MG cells.

Knockdown of PSMB4 decreases the migration and invasion in human glioblastoma cells

The migration and invasion abilities of PSMB4 knockdown human glioblastoma cells were assessed via wound healing and Transwell assays in LN229 and U87 MG cells. Decreased PSMB4 reduced the migration ability of LN229 and U87MG cells to $36 \%$ and $68 \%$ respectively, as compared with the control group, in the wound healing assay (Fig. 5A). In the Transwell migration assay, downregulated PSMB4 protein expression also markedly decreased the migration ability, by over 84\% in LN229 cells and 35\% in U87MG cells compared with that in the control group (Fig. 5B). In addition, the invasion ability of LN229 and U87MG cells was also significantly decreased by $80 \%$ and $30 \%$, respectively, as compared with the control 
Fig. 4. Long-term PSMB4 inhibition promotes apoptosis in GBM cells. LN229 and U87MG glioma cells with PSMB4 expression down-regulated for 96 hours. (A) Cell cycle expression was analyzed by FACS. The apoptotic cells of (B) LN229 and (C) U87MG were detected using Annexin V staining and flow cytometry analysis. The right panels show the percentage of the apoptotic cells. ${ }^{* * *} \mathrm{P}<0.001$ compared with the control group.

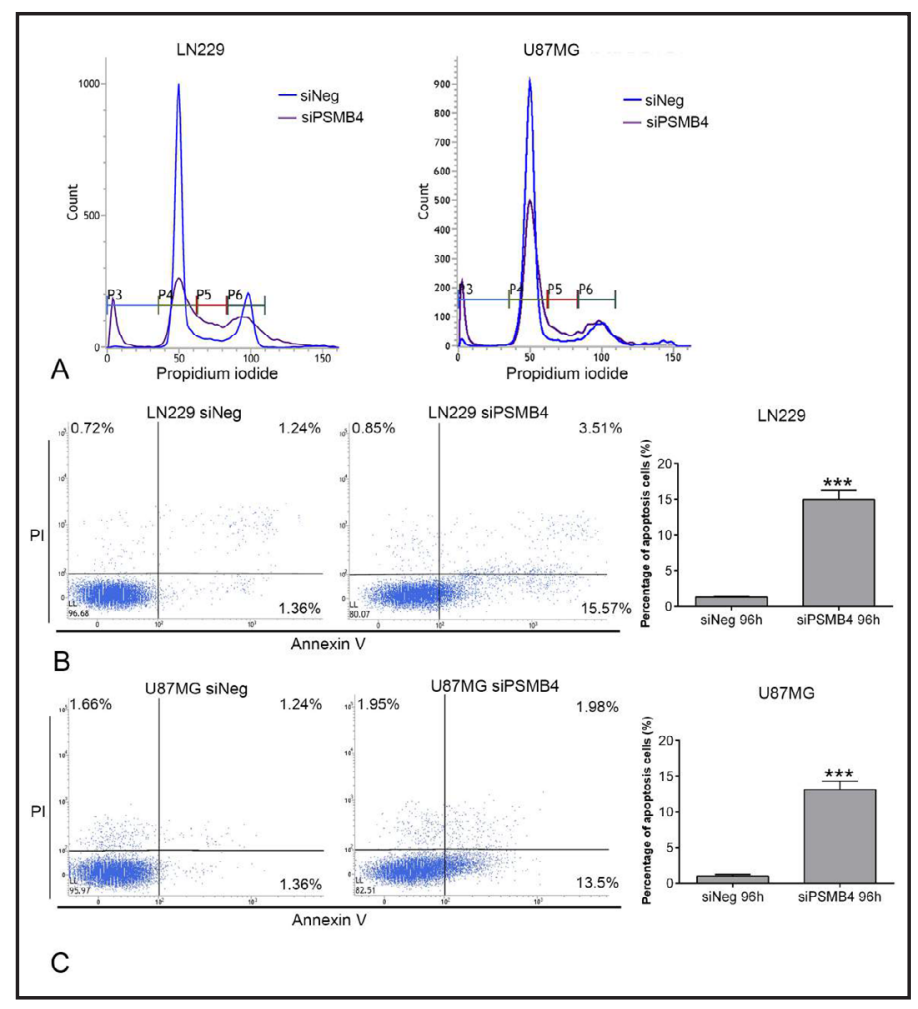

Fig. 5. Knockdown of PSMB4 decreases the ability of migration and invasion in human glioblastoma cells. The migration and invasion inhibition in PSMB4 knockdown LN229 and U87MG cells were analyzed using (A) wound healing migration assays, (B) Transwell migration assays and (C) Matrigel-coated Transwell invasion assays. The right panels show the quantitative analyses of the migration and invasion assays. $* * * \mathrm{p}<0.001$ compared with the control group.

group, after PSMB4 knockdown (Fig. 5C).

PSMB4 regulates the expression of adhesion-related and invasion-related proteins in human glioblastoma cells

To explore the mechanism underlying PSMB4's regulation of adhesion and invasion, the expression of adhesion-related and invasion-related proteins in LN229 cells with PSMB4 deficiency were measured. After compressing

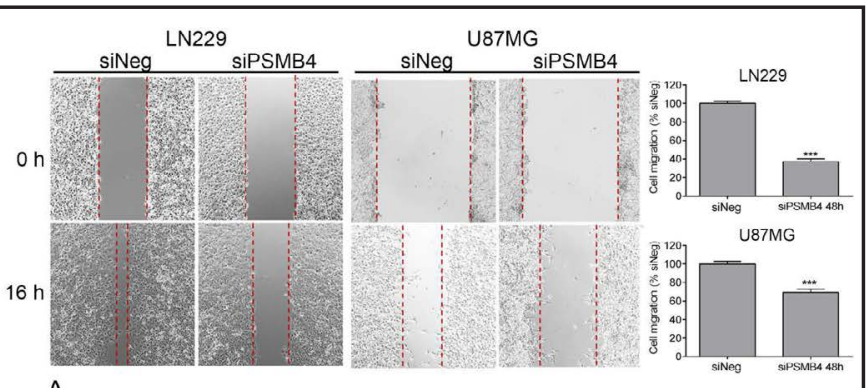
A
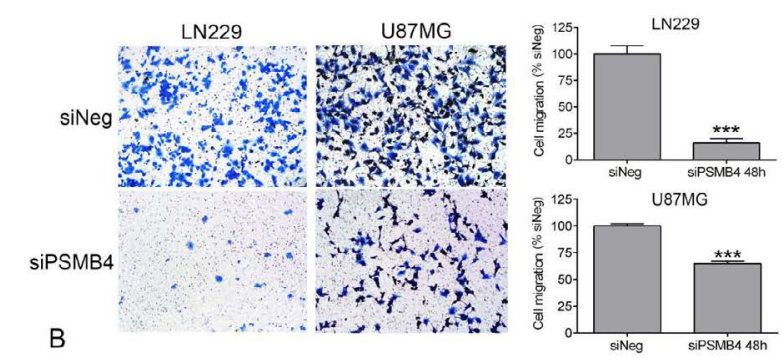

B

N229
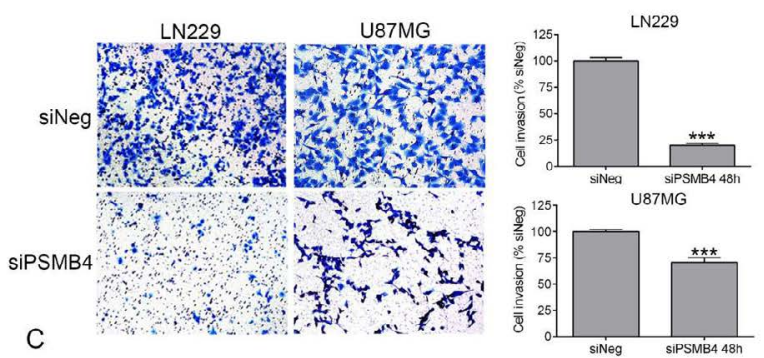
PSMB4 performance, the expres- 


\section{Cellular Physiology and Biochemistry}

Cell Physiol Biochem 2018;45:819-831

\begin{tabular}{l|l}
\hline DOI: $10.1159 / 000487174$ & (c) 2018 The Author(s). Published by S. Karger AG, Basel
\end{tabular}

Cheng et al.: PSMB4 Inhibition Reduces Glioblastoma Progression sion of p-FAK, p-paxillin, integrin $\beta 1$ and integrin $\beta 3$ were decreased (Fig. 6A). In addition, the invasion-related proteins, including MMP2, MMP9 and cathepsin B, were also decreased after the deficiency of PSMB4 (Fig. 6B). These results proved that the lack of PSMB4 decreased the adhesion-related and invasion-related proteins in LN229, thus potentially decreasing the adhesion and invasion abilities of human glioblastoma cells.

The effect of inhibiting PSMB4 expression in orthotropic human glioblastoma xenograft mouse models

Before intracranial implantation, LN229 cells labeled with luciferase were pretreated with non-target or PSMB4 siRNA for 48 hours to inhibit the expression of PSMB4. In the experimental end point in the animal model, the decreased PSMB4 expression combined with TMZ treatment significantly reduced tumor growth, as compared with that in the SiPSMB4, TMZ and vehicle control groups (Fig. 7A-B). The siPSMB4 and TMZ treatment groups partially restricted tumor growth. In addition, body weight in the SiPSMB4 alone and siPSMB4-TMZ combination groups was higher than that in the TMZ group (Fig. 7C). HE and IHC staining revealed that siPSMB4 and TMZ combination treatment inhibited Ki-67 expression, tumor growth, and the expression of phosphorylated FAK and MMP9 in the tumors (Fig. 7D). Thus, the siPSMB4 and TMZ combination treatment inhibited the progression and growth of glioblastoma cells in vivo.

\section{Discussion}

Over the past decade, various proteasome subunits have been found to be closely related with ovarian and skin cancer progression $[8,16,17]$. Through genomics-based RNAi
Fig. 6. Effect of PSMB4 on adhesion-related and invasion-related protein expression in human glioblastoma cells. After downregulating PSMB4 expression via siRNA in LN229 cells, adhesion-related and invasion-related protein expression was analyzed by western blotting for 24 and 48 hours, respectively. (A) Protein expression of PSMB4, p-FAK, FAK, p-paxillin, paxillin, integrin $\beta 1$ and integrin $\beta 3$ in LN229 cells. The right and lower panels show the quantitative analyses of western blotting for the proteins in (A). (B) Protein expression of MMP2, MMP9 and cathepsin B in LN229. The lower panels show the quantitative analyses of western blotting for the proteins in (B). GAPDH was used as an internal control. ${ }^{*} \mathrm{p}<0.05$; ${ }^{* *} \mathrm{p}<0.01 ;{ }^{* * *} \mathrm{P}<0.001$ compared with the control group. 


\section{Cellular Physiology and Biochemistry Published \begin{tabular}{l|l} 
DOI: 10.1159/000487174 & $\begin{array}{l}\text { ( ) } 2018 \text { The Author(s). Published by S. Karger AG, Basel } \\
\text { www.karger.com/cpb }\end{array}$
\end{tabular} \\ Cheng et al.: PSMB4 Inhibition Reduces Glioblastoma Progression}

Fig. 7. Downregulation of PSMB4 expression inhibits tumor progression in an orthotropic GBM xenograft mouse model. (A) The in vivo bioluminescence imaging data were examined in the vehicle control $(n=4), T M Z \quad(n=4)$, siPSMB4 $(n=5)$, siPSMB4+TMZ $(n=5)$ groups via the IVIS system. (B) The panels show the quantitative analyses of luminescence in LN229-Luc2 cells to identify the tumor status within 12 days. ${ }^{*} \mathrm{p}<0.05$; ${ }^{* * *} \mathrm{p}<0.001$ compared with the placebo group. ${ }^{\# \#} \mathrm{p}<0.01$ compared with the TMZ-treated group. ${ }^{\$} \mathrm{p}<0.01 ; \quad{ }^{\$ \$} \mathrm{p}<0.001$ compared with the siPSMB4 group. (C) Body weights. ${ }^{*} \mathrm{p}<0.05$ compared with the placebo group. (D) HE staining showed brain tumor tissue (white dotted line) relative to the surrounding normal brain tissue. Immunostaining of Ki-67, p-FAK and MMP9 in the brain tumor was performed. HE and immunostaining of brain and tumor sections were observed in 10 random fields for each group.

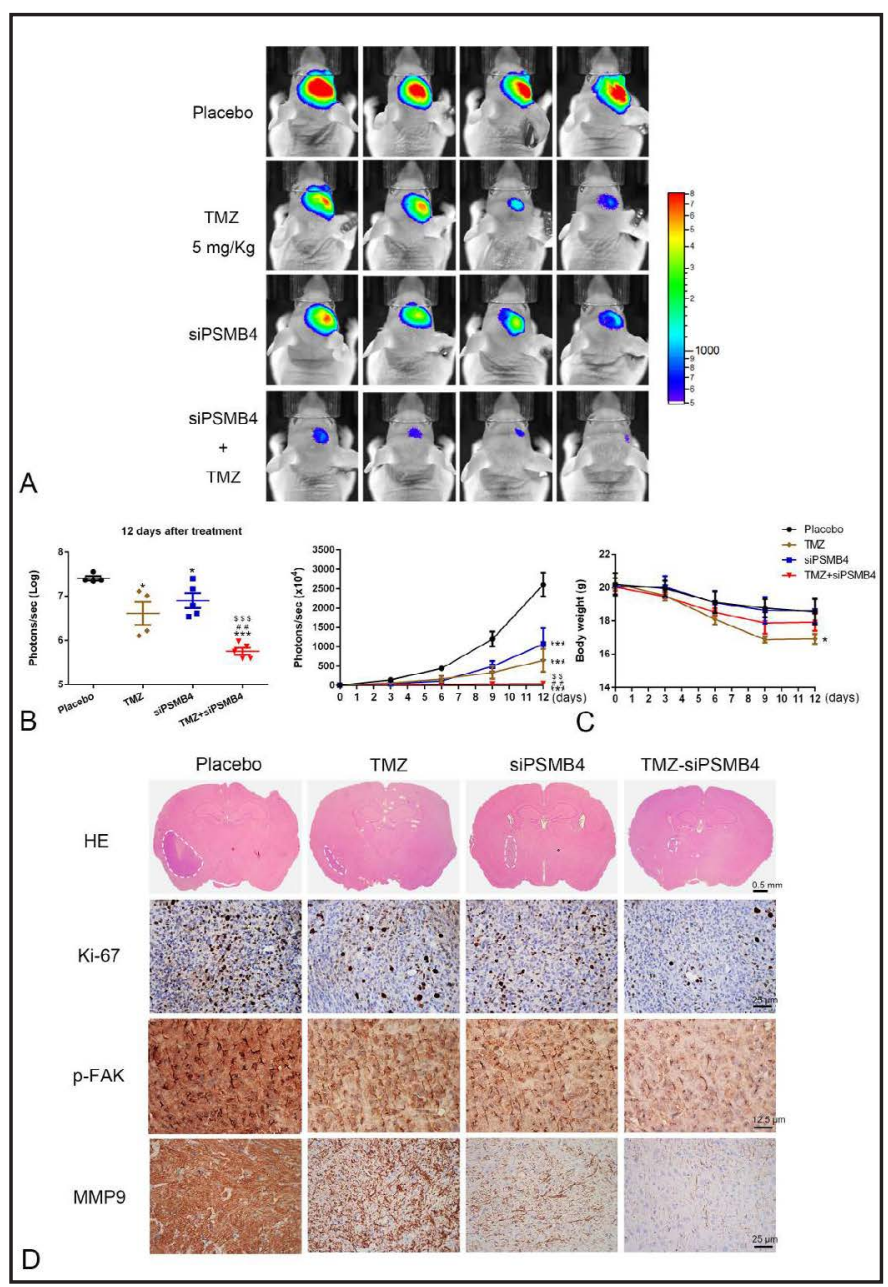

screens, it has been observed that $40 \%$ of enriched genes are components of the proteasome in breast and ovarian cancers [12]. Inhibition of proteasome activity has gradually become a promising strategy in cancer treatment $[18,19]$. PSMB4 was identified as a potential oncogene in human cancers and shows significant upregulation in breast, lung, ovarian and skin tumors $[8,10,12]$. In myeloma and ovarian cancer, downregulated PSMB4 expression results in decreased cell proliferation and NF- $\mathrm{KB}$ activity $[8,10]$. PSMB4 has also been proven to regulate the expression of NF- $\kappa \mathrm{B}$-mediated cell proliferation proteins, such as cyclin D1 and cyclin E in ovarian cancer $[8,10]$. In NIH/3T3 cells, the expression of PSMB4 promotes both anchorage-independent growth and tumorigenesis [12]. Furthermore, the upregulated expression of PSMB4 is positively correlated with poorer recurrence-free survival in breast cancer, metastasis-free survival in prostate cancer and lower overall survival in ovarian and prostate cancer $[11,12]$. In a recent study, PSMB4 has also been identified as a survival gene required for the proliferation of human glioblastoma cells [13]. However, there no previous studies have explored the effect and possible mechanism of PSMB4 on glioblastoma progression and invasion. To investigate the role of PSMB4 in glioma progression, we first examined the PSMB4 mRNA and protein expression in human glioma and normal brain tissues. Data from the GEO profiles indicated that, compared with low PSMB4 mRNA expression, high PSMB4 mRNA and protein expression predicts poor survival outcome. This study revealed that PSMB4 may serve as a marker of pathological grade and survival outcome.

The activation of MMP2 and MMP9 increases cancer cell invasion, proliferation and angiogenesis in human glioblastomas $[20,21]$. The composition with the correct assembly of the proteasome is critical to proteasome activity [22]. Several studies have indicated 
that a lack of PSMB4 disrupts the formation of the 20S proteasome, thus decreasing in proteasome activity, although PSMB4 does not have intrinsic hydrolytic activity [12, 22, 23]. Inhibition of proteasome activity also downregulates the expression of MMP2 and MMP9 in human non-small cell lung cancer $[24,25]$. In addition, recent studies have shown that MMP2 and MMP9 activation is also affected by cathepsin B [26, 27]. Increased cathepsin B expression is positively related to the migration and invasion ability of human glioblastoma [28]. Decreasing the expression of cathepsin B in human breast and lung cancer cells inhibits tumor metastasis and angiogenesis [29,30]. In the present study, the expression levels of MMP2, MMP9 and cathepsin B were decreased after PSMB4 knockdown in LN229 human glioblastoma cells. The downregulated PSMB4 might decrease the expression of MMP2, MMP9 and cathepsin B through deactivating the proteasome cascades.

Integrin $\beta 3$ is critical for cathepsin B-induced cancer progression in hepatocellular carcinoma [31]. Additionally, decreasing integrin $\beta 3$ expression prevents cathepsin B-induced activation of PI3K/Akt in HCC [31]. In the glioma cell line LN229, the expression levels of integrin $\beta 1$ and $\beta 3$ were decreased after reducing the PSMB4 expression. However, the underlying mechanisms among integrins, cathepsin B and PSMB4 are still unclear. The clustering of integrins also activates FAK and paxillin [32, 33]. The phosphorylation of FAK and paxillin catalyzes a cascade of kinases, including Rac, ERK and PI3K, which further enhance proliferation, cytoskeleton assembly and cell invasion [34, 35]. In our studies, reducing PSMB4 also inhibited the activation of FAK and paxillin. Therefore, on the basis of these findings, the increased expression of PSMB4 in glioma cells might facilitate GBM progression and invasion through activating the cathepsin $B$ and integrin/FAK/paxillin pathways.

TMZ, the first-line chemotherapeutic drug for GBM treatment, causes cancer cell death by inducing DNA double strand breaks [36, 37], but the human glioblastoma cell line LN229 has a high degree of resistance to TMZ [38, 39]. In the orthotopic xenograft mouse model, compared with the TMZ and siPSMB4 groups, the combination of TMZ-siPSMB4 group inhibited the progression of GBM tumors. These results indicated that PSMB4 inhibition in combination with TMZ might serve as an effective anti-tumor agent to target TMZ-resistant cells. In summary, our results provide evidence that the decreased PSMB4 expression inhibited the progression of LN229 glioma cells both in vivo and in vitro. Additionally, through deactivating cathepsin B, MMP2/9, integrin $\beta 1 / \beta 3$, FAK and paxillin, PSMB4 deficiency would further decrease the invasion of GBM cells, thus suggesting that targeting PSMB4 may be applied as an alternative therapeutic option to improve the efficacy of GBM treatment.

\section{Acknowledgements}

The authors would like to thank the members of the Tumour Microenvironment Laboratory for their input and critical reading of the manuscript.

The authors would like to thank the Dr. Dueng-Yuan Hueng, National Defense Medical Center, Taiwan, for kindly provided the human LN229 and U87MG glioblastoma cell lines and GBM8401 primary cell line. This work was supported by the Ministry of Science and Technology, Taiwan (grant number: 103-2320-B-016-003-MY3 and 106-2320-B-016-015).

\section{Disclosure Statement}

The authors have nothing to disclose. All authors have made a substantial contribution to this work, have read the manuscript and approve of it. None of the authors have any financial and personal relationships with other people or organizations that could inappropriately influence this work. 


\section{Cellular Physiology Cell Physiol Biochem 2018;45:819-831 and Biochemistry DOI: 10.1159/000487174 2018 Published 2018 The Author(s). Published by S. Karger AG, Basel

\section{References}

1 Bleeker FE, Molenaar RJ, Leenstra S: Recent advances in the molecular understanding of glioblastoma. J Neurooncol 2012;108:11-27.

-2 Sehgal A: Molecular changes during the genesis of human gliomas. Semin Surg Oncol 1998;14:3-12.

-3 Bredel M, Piribauer M, Marosi C, Birner P, Gatterbauer B, Fischer I, Strobel T, Rossler K, Budka H, Hainfellner JA: High expression of DNA topoisomerase IIalpha and Ki-67 antigen is associated with prolonged survival in glioblastoma patients. Eur J Cancer 2002;38:1343-1347.

-4 Johnson DR, O'Neill BP: Glioblastoma survival in the United States before and during the temozolomide era. J Neurooncol 2012;107:359-364.

-5 Van Meir EG, Hadjipanayis CG, Norden AD, Shu HK, Wen PY, Olson JJ: Exciting new advances in neurooncology: the avenue to a cure for malignant glioma. CA Cancer J Clin 2010;60:166-193.

6 Ostrom QT, Bauchet L, Davis FG, Deltour I, Fisher JL, Langer CE, Pekmezci M, Schwartzbaum JA, Turner MC, Walsh KM, Wrensch MR, Barnholtz-Sloan JS: The epidemiology of glioma in adults: a "state of the science" review. Neuro Oncol 2014;16:896-913.

7 Nothwang HG, Tamura T, Tanaka K, Ichihara A: Sequence analyses and inter-species comparisons of three novel human proteasomal subunits, HsN3, HsC7-I and HsC10-II, confine potential proteolytic active-site residues. Biochim Biophys Acta 1994;1219:361-368.

8 Zheng P, Guo H, Li G, Han S, Luo F, Liu Y: PSMB4 promotes multiple myeloma cell growth by activating NFkappaB-miR-21 signaling. Biochem Biophys Res Commun 2015;458:328-333.

-9 Cui F, Wang Y, Wang J, Wei K, Hu J, Liu F, Wang H, Zhao X, Zhang X, Yang X: The up-regulation of proteasome subunits and lysosomal proteases in hepatocellular carcinomas of the HBx gene knockin transgenic mice. Proteomics 2006;6:498-504.

10 Liu R, Lu S, Deng Y, Yang S, He S, Cai J, Qiang F, Chen C, Zhang W, Zhao S, Qian L, Mao G, Wang Y: PSMB4 expression associates with epithelial ovarian cancer growth and poor prognosis. Arch Gynecol Obstet 2016;293:1297-1307.

11 Zapatero A, Morente M, Nieto S, Martin de Vidales C, Lopez C, Adrados M, Arellano R, Artiga MJ, GarciaVicente F, Herranz LM, Leaman O: Predictive value of PAK6 and PSMB4 expression in patients with localized prostate cancer treated with dose-escalation radiation therapy and androgen deprivation therapy. Urol Oncol 2014;32:1327-1332.

12 Lee GY, Haverty PM, Li L, Kljavin NM, Bourgon R, Lee J, Stern H, Modrusan Z, Seshagiri S, Zhang Z, Davis D, Stokoe D, Settleman J, de Sauvage FJ, Neve RM: Comparative oncogenomics identifies PSMB4 and SHMT2 as potential cancer driver genes. Cancer Res 2014;74:3114-3126.

13 Thaker NG, Zhang F, McDonald PR, Shun TY, Lewen MD, Pollack IF, Lazo JS: Identification of survival genes in human glioblastoma cells by small interfering RNA screening. Mol Pharmacol 2009;76:1246-1255.

14 Hueng DY, Tsai WC, Chiou HY, Feng SW, Lin C, Li YF, Huang LC, Lin MH: DDX3X Biomarker Correlates with Poor Survival in Human Gliomas. Int J Mol Sci 2015;16:15578-15591.

15 Hueng DY, Lin GJ, Huang SH, Liu LW, Ju DT, Chen YW, Sytwu HK, Chang C, Huang SM, Yeh YS, Lee HM, Ma HI: Inhibition of Nodal suppresses angiogenesis and growth of human gliomas. J Neurooncol 2011;104:21-31.

-16 Kisselev AF, Goldberg AL: Proteasome inhibitors: from research tools to drug candidates. Chem Biol 2001;8:739-758.

17 Zhang P, Bi C, Schmitt SM, Li X, Fan Y, Zhang N, Dou QP: Metal-based 2, 3-indolinedione derivatives as proteasome inhibitors and inducers of apoptosis in human cancer cells. Int J Mol Med 2014;34:870-879.

18 Ruschak AM, Slassi M, Kay LE, Schimmer AD: Novel proteasome inhibitors to overcome bortezomib resistance. J Natl Cancer Inst 2011;103:1007-1017.

19 Shen M, Schmitt S, Buac D, Dou QP: Targeting the ubiquitin-proteasome system for cancer therapy. Expert Opin Ther Targets 2013;17:1091-1108.

20 Bello L, Lucini V, Carrabba G, Giussani C, Machluf M, Pluderi M, Nikas D, Zhang J, Tomei G, Villani RM, Carroll RS, Bikfalvi A, Black PM: Simultaneous inhibition of glioma angiogenesis, cell proliferation, and invasion by a naturally occurring fragment of human metalloproteinase-2. Cancer Res 2001;61:8730-8736.

21 Sun C, Wang Q, Zhou H, Yu S, Simard AR, Kang C, Li Y, Kong Y, An T, Wen Y, Shi F, Hao J: Antisense MMP-9 RNA inhibits malignant glioma cell growth in vitro and in vivo. Neurosci Bull 2013;29:83-93.

22 Murata S, Yashiroda H, Tanaka K: Molecular mechanisms of proteasome assembly. Nat Rev Mol Cell Biol 2009;10:104-115. 


\section{Cellular Physiology Cell Physiol Biochem 2018;45:819-831

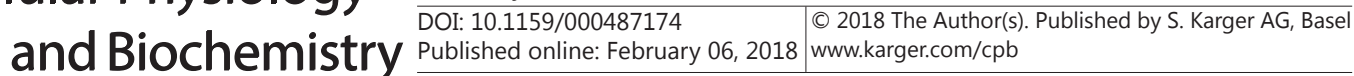

23 Hirano Y, Kaneko T, Okamoto K, Bai M, Yashiroda H, Furuyama K, Kato K, Tanaka K, Murata S: Dissecting beta-ring assembly pathway of the mammalian 20S proteasome. EMBO J 2008;27:2204-2213.

-24 Karthik S, Sankar R, Varunkumar K, Ravikumar V: Romidepsin induces cell cycle arrest, apoptosis, histone hyperacetylation and reduces matrix metalloproteinases 2 and 9 expression in bortezomib sensitized nonsmall cell lung cancer cells. Biomed Pharmacother 2014;68:327-334.

25 van Rijt SH, Bolukbas DA, Argyo C, Datz S, Lindner M, Eickelberg O, Konigshoff M, Bein T, Meiners S: Protease-mediated release of chemotherapeutics from mesoporous silica nanoparticles to ex vivo human and mouse lung tumors. ACS Nano 2015;9:2377-2389.

-26 Schuler PJ, Bendszus M, Kuehnel S, Wagner S, Hoffmann TK, Goldbrunner R, Vince GH: Urokinase plasminogen activator, uPAR, MMP-2, and MMP-9 in the C6-glioblastoma rat model. In vivo 2012;26:571576.

27 Zhao Y, Lyons CE, Jr., Xiao A, Templeton DJ, Sang QA, Brew K, Hussaini IM: Urokinase directly activates matrix metalloproteinases-9: a potential role in glioblastoma invasion. Biochem Biophys Res Commun 2008;369:1215-1220.

28 Vasiljeva 0, Turk B: Dual contrasting roles of cysteine cathepsins in cancer progression: apoptosis versus tumour invasion. Biochimie 2008;90:380-386.

29 Withana NP, Blum G, Sameni M, Slaney C, Anbalagan A, Olive MB, Bidwell BN, Edgington L, Wang L, Moin K, Sloane BF, Anderson RL, Bogyo MS, Parker BS: Cathepsin B inhibition limits bone metastasis in breast cancer. Cancer Res 2012;72:1199-1209.

-30 Vasiljeva O, Papazoglou A, Kruger A, Brodoefel H, Korovin M, Deussing J, Augustin N, Nielsen BS, Almholt K, Bogyo M, Peters C, Reinheckel T: Tumor cell-derived and macrophage-derived cathepsin B promotes progression and lung metastasis of mammary cancer. Cancer Res 2006;66:5242-5250.

-31 Xu ZZ, Xiu P, Lv JW, Wang FH, Dong XF, Liu F, Li T, Li J: Integrin alphavbeta3 is required for cathepsin B-induced hepatocellular carcinoma progression. Mol Med Rep 2015;11:3499-3504.

-32 Ware ML, Berger MS, Binder DK: Molecular biology of glioma tumorigenesis. Histol Histopathol 2003;18:207-216.

33 Shan Y, Yu L, Li Y, Pan Y, Zhang Q Wang F, Chen J, Zhu X: Nudel and FAK as antagonizing strength modulators of nascent adhesions through paxillin. PLoS Biol 2009;7:e1000116.

-34 Obara S, Nakata M, Takeshima H, Kuratsu J, Maruyama I, Kitajima I: Inhibition of migration of human glioblastoma cells by cerivastatin in association with focal adhesion kinase (FAK). Cancer Lett 2002;185:153-161.

-35 Ross RS: Molecular and mechanical synergy: cross-talk between integrins and growth factor receptors. Cardiovasc Res 2004;63:381-390.

-36 Stupp R, Mason WP, van den Bent MJ, Weller M, Fisher B, Taphoorn MJ, Belanger K, Brandes AA, Marosi C, Bogdahn U, Curschmann J, Janzer RC, Ludwin SK, Gorlia T, Allgeier A, Lacombe D, Cairncross JG, Eisenhauer E, Mirimanoff RO, European Organisation for R, Treatment of Cancer Brain T, Radiotherapy G, National Cancer Institute of Canada Clinical Trials G: Radiotherapy plus concomitant and adjuvant temozolomide for glioblastoma. N Engl J Med 2005;352:987-996.

-37 Newlands ES, Blackledge GR, Slack JA, Rustin GJ, Smith DB, Stuart NS, Quarterman CP, Hoffman R, Stevens MF, Brampton MH, et al.: Phase I trial of temozolomide (CCRG 81045: M\&B 39831: NSC 362856). Br J Cancer 1992;65:287-291.

-38 Cuperlovic-Culf M, Touaibia M, St-Coeur PD, Poitras J, Morin P, Culf AS: Metabolic Effects of Known and Novel HDAC and SIRT Inhibitors in Glioblastomas Independently or Combined with Temozolomide. Metabolites 2014;4:807-830.

-39 Cheng Y, Sk UH, Zhang Y, Ren X, Zhang L, Huber-Keener KJ, Sun YW, Liao J, Amin S, Sharma AK, Yang JM: Rational incorporation of selenium into temozolomide elicits superior antitumor activity associated with both apoptotic and autophagic cell death. PLoS One 2012; 7 :e35104. 